\title{
Identificación de serovares de Leptospira sp. en búfalos de Corrientes, Argentina
}

\author{
Jacobo, R.A. ${ }^{\text {; }}$ Cipolini, M.F.'; Martínez, D.E. ${ }^{1}$; Dellamea, M. ${ }^{\text {; }}$ Draghi, M.G. ${ }^{2}$ \\ ${ }^{1}$ Cátedra Enfermedades Infecciosas, Facultad de Ciencias Veterinarias, UNNE, Sargento Cabral 2139, \\ Corrientes (3400), Argentina. Tel/fax: 03783-425753. ${ }^{2}$ INTA Mercedes, Corrientes, Argentina. \\ E-mail: enfinf@vet.unne.edu.ar.
}

\begin{abstract}
Resumen
Jacobo, R.A.; Cipolini, M.F.; Martínez, D.E.; Dellamea, M.; Draghi, M.G.: Identificación de serovares de Leptospira sp. en búfalos de Corrientes, Argentina. Rev. vet. 20: 2, 126-127, 2009. Con el objetivo de efectuar los primeros diagnóstico serológicos para determinar los serovares prevalentes de Leptospira sp. en búfalos, se realizó un muestreo en animales pertenecientes a siete rodeos bubalinos de la región noroeste de la Provincia de Corrientes, Argentina $(\mathrm{n}=550$ ). Los sueros sanguíneos fueron procesados por microaglutinación con antígenos vivos mantenidos en cultivos especiales (MAT). Se identificaron los serovares tarassovi y wolfii.
\end{abstract}

Palabras clave: búfalo, leptospirosis, serovares, Argentina.

\begin{abstract}
Jacobo, R.A.; Cipolini, M.F.; Martínez, D.E.; Dellamea, M.; Draghi, M.G.: Identification of Leptospira sp. serovars in buffalos from Corrientes, Argentina. Rev. vet. 20: 2, 126-127, 2009. With the purpose to obtain serologic data regarding prevalence of Leptospira $s p$. serovars of buffaloes from northwest Corrientes, Argentina, blood samples of animals $(\mathrm{n}=500)$ from seven ranches were analyzed. Sera were processed with the MAT routine technique. The tarassovi as well as wolfi serovars were identified.
\end{abstract}

Key words: buffalo, leptospirosis, serovars, Argentina.

\section{INTRODUCCIÓN}

La producción bubalina en la Provincia de Corrientes se ve favorecida por las condiciones topográficas y ecológicas de sus campos, considerados marginales para el ganado bovino, que permiten a los búfalos adaptarse y expresar la elevada performance propia de la especie ${ }^{2,10}$. Ello indujo que los productores incorporen esta especie de ganado exótico a sus empresas, con miras a la explotación tambera y elaboración de derivados como el queso mozzarella. En otros casos se destina a la obtención de terneros o novillos para ubicarlos en los mercados de carnes ${ }^{10}$.

Las cualidades productivas y el componente sanitario son aspectos importantes a ser tenidos en cuenta en la producción bubalina, especialmente con referencia a la calidad de la leche y a las enfermedades de fuerte impacto económico y mayor difusión en Argentina. Dentro del aspecto productivo, puntualmente la calidad de la leche, nuestro grupo de trabajo está investigando su composición y las características de sus derivados, especialmente quesos.

Recibido: 5 agosto 2009 / Aceptado: 14 octubre 2009
En cuanto a la sanidad, en Latinoamérica no abundan reportes que describan la incidencia y prevalencia de enfermedades del búfalo. En países del viejo continente el ganado bubalino reveló seropositividad para enfermedades como brucelosis $(2,6 \%)$, leucosis enzoótica bovina $(1,3 \%)$, rinotraqueítis infecciosa bovinaIBR $(40 \%)$ y leptospirosis $(39 \%)^{8,9}$.

En Argentina el conocimiento de la transmisión, distribución e impacto de las distintas enfermedades infecciosas del búfalo es aún incipiente. Para nuestra zona de influencia se efectuaron comunicaciones sobre mastitis y alteraciones de la glándula mamaria, duración de aglutininas vacunales generadas por la vacuna C19 contra brucelosis e identificación de animales infectados con los agentes responsables del complejo tristeza del bovino ${ }^{6}$.

La especie bubalina, mantenida habitualmente en campos bajos y anegadizos en zonas de clima subtropical, con temperatura y humedad elevadas, está expuesta a distintos gérmenes ambientales, tal el caso de las leptospiras. Por tal motivo y teniendo en cuenta la importancia de este agente infeccioso en la producción y en la salud pública, se consideró importante conocer su distribución en el búfalo. El objetivo del trabajo fue 
determinar los serovares prevalentes de Leptospira $s p$. en búfalos de la región noroeste de la Provincia de Corrientes.

\section{MATERIAL Y MÉTODOS}

El muestreo se llevó a cabo en 550 búfalos de ambos sexos, pertenecientes a 7 rodeos ubicados en los departamentos de General Paz, Empedrado, San Cosme, San Luis del Palmar e Itatí, todos ubicados en la zona noroeste de la Provincia de Corrientes, Argentina. Al no contarse con trabajos previos sobre diagnóstico de leptospirosis que permitan conocer su prevalencia en cada establecimiento, por medio del sistema aleatorio sistemático sin reposición se seleccionó para muestreo el 20\% de los animales mayores de un año.

La sangre se obtuvo por venopunción yugular. El suero se procesó por medio de la técnica de microaglutinación (MAT) con antígenos vivos mantenidos en cultivos especiales ${ }^{1,4}$. Las muestras fueron procesadas en laboratorios del INTA Mercedes (Corrientes, Argentina), por contar con los serovares prevalentes en la región, capaces de afectar al ganado bovino (tarassovi, pomona, wolfii, grippotyphosa) ${ }^{3}$.

Se prepararon diluciones del suero de 1:25 hasta 1:3200 con solución salina fisiológica tamponada. A 0,2 $\mathrm{ml}$ de cada dilución del suero se agregó $0,2 \mathrm{ml}$ de antígeno, luego los tubos se agitaron y se incubaron a $30^{\circ} \mathrm{C}$ por $3 \mathrm{~h}$ para su observación final. La misma se realizó depositando en un portaobjetos una gota de cada tubo y examinándola en microscopio de campo oscuro, a través de un objetivo de pequeño aumento y oculares $15 x$, sin cubreobjetos. El grado de aglutinación se interpretó como positivo o negativo, reconociéndose una reacción como positiva si tenía $50 \%$ o más de las leptospiras aglutinadas.

\section{RESULTADOS Y DISCUSIÓN}

Cabe señalar que la zona de trabajo se caracteriza por contar con un importante número de lagunas, bañados y arroyos, lo cual le confiere una de las condiciones básicas que requiere Leptospira sp. para permanecer en el medio, esto es: aguas estancadas o de corriente lenta, así como temperaturas entre 13 y $32^{\circ} \mathrm{C}$.

Para el total de las muestras, el test MAT reveló positividad para los serovares tarassovi y wolfi del agrupamiento o cepa interrogans, en la dilución 1:1200.
Los serovares identificados no son coincidentes con los que habitualmente infectan al bovino a nivel nacional, tal el caso de las variedades de hardjo, pomona y grippotyphosa ${ }^{8}$. Pero sí son los mismos serovares oportunamente identificados en bovinos de la Provincia de Corrientes $^{3}$, a los que se suman pomona y ballum. Las variedades aquí encontradas son coincidentes con las halladas en Brasil ${ }^{5,7}$.

Agradecimiento. Al personal del Laboratorio de Diagnóstico de la Estación Experimental Agropecuaria del INTA de Mercedes (Corrientes) por realizar los trabajos de laboratorio.

\section{REFERENCIAS}

1. Asociación Argentina de Veterinarios de Laboratorio de Diagnóstico. 1994. Manual de Leptospirosis, Ed. Comisión Científica Permanente sobre Leptospirosis, Buenos Aires, $16 \mathrm{p}$.

2. Crudelli GA, Patiño EM, Cedrés JF, Maldonado P, Pellerano GS. 2002. Buffalo breeding in Argentina. J Buffalo Sci Tech 3: 7-17.

3. Draghi MG. 2000. Leptospirosis. Boletín Noticias y Comentarios INTA $\mathrm{N}^{\mathrm{o}} 338$, Mercedes, Corrientes (Argentina), $6 \mathrm{p}$.

4. Francois S. 2008. Test de microaglutinación (MAT). Anales XVII Reunión AAVLD, Santa Fe (Argentina), p. 5-6.

5. Girio RJ. 1984. Estudio comparativo de quatro cepas de leptospiras aptogénicas utilizadas em teste de triagem para o diagnóstico sorológico da leptospirose em búfalos Tesis de Maestrado, Univ. Sao Paulo (Brasil), 188 p.

6. Jacobo RA, Cipolini MF, Storani CA, Martínez DE, Martínez EI. 2004. Infección con el complejo tristeza del bovino en búfalos. Rev Med Vet 85: 203-204.

7. Langoni H, Del Fava C, Abral KG, Silva AV, Chagas SA. 1997. Epidemiological survey on anti-leptospire agglutinins in buffaloes from Vale do Ribeira (Brazil). Proceeding of 5th World Buffalo Congress, Caserta (Italy), $\mathrm{p}$ 622-625.

8. Rosetti CA. 1999. Leptospirosis en las especies de producción. Rev Med Vet 80: 412-413.

9. Tealdo M. 2008. Leptospirosis, epidemiología. Anales XVII Reunión AAVLD, Santa Fe (Argentina), p.19-20.

10. Zava M. 2000. Búfalos en el mundo y en Argentina. Memorias I Simposio de Búfalos del Mercosur, Corrientes (Argentina), p. 15-22. 